The following paper is the accepted version published in Imaging Systems and Techniques (IST), 2012 IEEE International Conference on. The published paper can be found using the following link (http://ieeexplore.ieee.org/xpls/abs_all.jsp?arnumber=6295591\&tag=1).

Paper

DOI (10.1109/IST.2012.6295591). 


\title{
Mitigating the Effect of Optical Back-scatter in Multispectral Underwater Imaging
}

\author{
Halleh Mortazavi ${ }^{1}$, John P. Oakley ${ }^{1}$ and Braham Barkat ${ }^{2}$ \\ ${ }^{1}$ Signal, Sensing and Image Processing (SISP), School of Electrical and Electronic Eng, The \\ University of Manchester, UK \\ ${ }^{2}$ Department of Electrical Eng, The Petroleum Institute, Abu Dhabi, UAE \\ E-mail: Halleh.Mortazavi@manchester.ac.uk, \\ j.oakley@manchester.ac.uk, bbarkat@pi.ac.ae
}

\begin{abstract}
Multispectral imaging is a very useful technique for extracting information from the underwater world. However, optical back-scatter changes the intensity value in each spectral band and this distorts the estimated spectrum. In this work, a filter is used to detect the level of optical back-scatter in each spectral band from a set of multispectral images. Extraction of an underwater object spectra can be done by subtracting the estimated level of optical back-scatter and scaling the remainder in each spectral band from the captured image in the corresponding band. An experiment is designed to show the performance of the proposed filter for correcting the set of multispectral underwater images and recovering the pixel spectra. The multispectral images are captured by a B/W CCD digital camera with a fast tunable liquidcrystal filter in 33 narrow spectral bands in clear and different levels of turbid water. Reference estimates for the optical back-scatter spectra are found by comparing a clear and degraded set of multispectral images. The accuracy and consistency of the proposed method, the extended Oakley-Bu cost function, is examined by comparing the estimated values with the reference level of optical back-scatter spectrum. The same comparison is made for the simple estimation approach. The results show that the simple method is not reliable and fail to estimate the level of optical back-scatter spectrum accurately. The results of processing experimental images in turbid water show that the effect of optical back-scatter can be mitigated in the image of each spectral band and, as a result, the spectra of the object can be recovered. However, for very high level of turbid water the recovery is limited because of the effect of extinction.
\end{abstract}

Keywords: Optical back-scatter, Underwater image, Multispectral image, Spectral reflectance 


\section{Introduction}

Underwater imaging is widely used in different underwater applications to extract information, such as marine biology, underwater target and mine detection. Multispectral imaging provides additional information over RGB images, because multispectral imaging provides an estimate for the scene reflectance spectrum at each image pixel by measuring intensities at several spectral bands. Accurate estimation spectral reflectance helps better surveying and understanding of underwater life. However, detecting accurate spectral features of a scene from an underwater multispectral image is problematic.

Optical scattering causes underwater images to be degraded in terms of both overall scattering and spatial resolution. Schechner and Karpel (Schechner \& Karpel 2005) suggest that the contrast loss is the significant problem. Moreover, it is shown experimentally that the contrast loss is the dominant degradation problem in comparison with resolution loss (Mortazavi 2010). The cause of contrast loss is due to the effect of optical back-scatter, which is the light scattered by suspended particles towards the camera. Optical back-scatter adds to the pixel intensity and as a result causes a change in the measured pixel reflectance spectrum. The magnitude of this extra component depends on different parameters, such as wavelength, the density and size of suspended particles in water and also the length of optical path. Therefore, the recorded reflectance spectrum of an underwater scene is not reliable. The effect of optical backscatter has been studied in underwater multispectral imaging (Holden \& LeDrew 2002, Lubin et al. 2001), but no general solution is given.

Previous studies have shown it is possible to compensate for the effect of optical backscatter (Oakley \& Bu 2007, Mortazavi \& Oakley 2007, Schechner \& Karpel 2005), but these studies are concerned with recovery of pixel intensity in RGB images. This work focuses on mitigating the effect of optical back-scatter in multispectral images. The aim is to recover a reflectance spectrum for each pixel. 
This paper is organised as follows. Firstly, a simple model of a degraded image pixel in turbid water in visible wavelengths is explained. Also, the recovery of the pixel intensity and then calculation of the pixel reflectance are explained. A method of estimating optical backscatter for a degraded image pixel is described. A water tank experiment is used to provide a controlled imaging situation for which a clear reference spectrum is available. A routine is explained to find the actual reference value of optical back-scatter spectrum using the set of clear and degraded multispectral images. The experiment and the results are presented in Sections 4 and 5, and finally the conclusion is given in Section 6.

\section{Image Model in Turbid Water}

\subsection{Simple Image Model}

A model of an image pixel in a specific wavelength $(\lambda)$ and in a turbid medium, $I_{\lambda}(x, y)$, is represented by (1) (Schechner \& Averbuch 2007). This is known as the additive model in the literature (Oakley \& Bu 2007).

$$
I_{\lambda}(x, y)=b_{\lambda}(x, y)+a_{\lambda}(x, y) F_{\lambda}(x, y)
$$

where $b_{\lambda}(x, y)$ is the optical back-scatter, $a_{\lambda}(x, y)$ is the attenuation factor (representing the generalised Beer-Lambert extinction for turbid media) and $F_{\lambda}(x, y)$, the scene intensity in clear condition at a specific wavelength and spatial pixel position. Recovery of the intensity of a pixel, $\hat{I}_{\lambda}(x, y)$, can be achieved by rearranging (1).

$$
\hat{I}_{\lambda}(x, y)=\frac{I_{\lambda}(x, y)-b_{\lambda}(x, y)}{a_{\lambda}(x, y)}
$$




\subsection{Spectral Reflectance Recovery}

The aim is to estimate spectral reflectance of each pixel under the assumption that the surface is Lambertian. In general the way in which surfaces reflect light depends on the angles of incidence and reflection and is specified by the Bi-Directional Reflectance Function. However in this experiment the scattering particles cause the illumination to become diffuse and the directional dependency of reflectance is not relevant. Unlike pixel intensity, surface spectral reflectance is an inherent property of an object and is independent of other parameters like time, location and illumination. Spectral reflectance can not be measured directly and instead must be calculated. The conversion of pixel intensity to reflectance can be done by scaling the pixel intensity spectrum by the spectral radiance of a white reference (Peddle et al. 2001). Therefore, by scaling the intensity spectrum of the recovered pixel with a white reference spectrum the recovered spectral reflectance of an image pixel can be obtained.

\section{Method}

\subsection{Proposed Method: Extended Oakley-Bu Cost Function Filter}

The proposed method is based on (2), by which the contrast loss can be mitigated by subtracting optical back-scatter, $b_{\lambda}(x, y)$, from the degraded underwater image and then multiplying the image by a scaling parameter, $1 / a_{\lambda}(x, y)$. The key issue is how to estimate $b_{\lambda}(x, y)$. For the purpose of simplifying the notation, the abbreviation form of $b_{\lambda}(x, y)$, $a_{\lambda}(x, y)$ and $I_{\lambda}(x, y)$ which are respectively $b_{\lambda}, a_{\lambda}$ and $I_{\lambda}$, are used for the rest of the paper.

The distribution of optical back-scatter is not stationary (Schechner \& Karpel 2004). Optical back-scatter follows the scene illumination spatial distribution and is independent of the scene content (Mortazavi \& Oakley 2007). The objective in this work is to estimate the pattern of illumination. Once this is achieved the underlying reflectance factor can be determined. The spatial distribution of scene illumination depends on the light source, which 
provides the required illumination in underwater imaging. The part of the scene that is directly in front of the beam, is illuminated more and the surroundings are less illuminated. This produces a hump at the part with high illumination. As a result, it can be concluded that the scene illumination distribution can be obtained by applying a low pass (averaging) filter to the image, either in time or space. For that reason $b_{\lambda}$ can be represented by (3).

$$
b_{\lambda}=\gamma \bar{I}_{\lambda},
$$

where $\bar{I}_{\lambda}$ is the smooth version of the image intensity and $\gamma$ is the coefficient that shows the contribution of optical back-scatter in image intensity. To estimate $b_{\lambda}$, the smooth version of the image and a constant parameter, $\gamma$, are required. The smooth version of the image is obtained using a recursive Gaussian filter with standard deviation of a relatively large value of $\sigma_{B}$ (Mortazavi \& Oakley 2007, Oakley \& Bu 2007). Hence, $\gamma$ is the only unknown parameter.

Oakley \& Bu (2007) introduced a new statistical method, the Oakley-Bu cost function, to detect a constant level of optical back-scatter in a degraded image, which is taken in adverse atmosphere condition such as fog or haze. The Oakley-Bu cost function is extended here to a variable spatial distribution of optical back-scatter. It is shown mathematically in Appendix A that the extended Oakley-Bu cost function has a minimum value at $\gamma$. Therefore, the value of $\gamma$ can be estimated by the extended Oakley-Bu cost function, which is given by (4).

$$
S_{\lambda}(\gamma)=\frac{1}{P} \sum_{p=1}^{P}\left(\frac{I_{\lambda}(p)-\bar{I}_{\lambda}^{c f}(p)}{\bar{I}_{\lambda}^{c f}(p)-\gamma \bar{I}_{\lambda}(p)}\right)^{2} \cdot \exp \left(\frac{1}{P} \sum_{p=1}^{P} \ln \left(\bar{I}_{\lambda}^{c f}(p)-\gamma \bar{I}_{\lambda}(p)\right)^{2}\right)
$$

where $p$ represents each image pixel in spatial position of $(x, y), P$ is the total number of pixels, $\lambda$ is the specific wavelength, $I_{\lambda}$ is the degraded image, $\bar{I}_{\lambda}^{c f}$ is the smooth version of the image, which is calculated by a recursive Gaussian filter with filter parameter $\sigma_{c f}$, and $\bar{I}_{\lambda}$ is the smooth version of the image, which is calculated by a recursive Gaussian filter with filter 
parameter $\sigma_{B}$. The scaling of image, $1 / a_{\lambda}(x, y)$, is to recover for the effect of extinction, after the degraded image is compensated for the effect of optical back-scatter. The value of $a_{\lambda}(x, y)$ is always less than unity and so multiplication by $1 / a_{\lambda}(x, y)$ effectively stretches the histogram of the image.

\subsection{Simple Approach: Minimum Pixel Filter}

Equation (1) implies that optical back-scatter is an extra term added to an image pixel intensity. Therefore, if we assume that the image pixel in clear condition is from a dark object, $F_{\lambda}(x, y) \approx 0$, then the value of this dark image pixel in a turbid medium should represent for the level of optical back-scatter, $I_{\lambda}(x, y)=b_{\lambda}(x, y)$, (Mortazavi 2010).

This method uses a rank filter to take the minimum pixel value from a set of degraded sample pixels of the same level of optical back-scatter as the value of $b_{\lambda}(x, y)$ parameter. These sample of pixels can be from one pixel position at different images with same level of optical back-scatter and different scene content.

\section{Experiment}

\subsection{Experimental Procedure}

A water tank experiment is designed to provide an imaging system in a controlled environment, that captures underwater multispectral images from different scene contents in both turbid and clear water conditions. In multispectral images, the wavelength can be assumed constant for each monochrome image in narrow spectral band. Therefore, by using multispectral images the parameter wavelength is kept constant. The clear water condition is used to obtain the pixel reflectance spectrum in a non scattering condition (no optical backscatter). The level of optical back-scatter in turbid water is kept constant during imaging by keeping the wavelength, particle size and density and length of the optical path constant. 


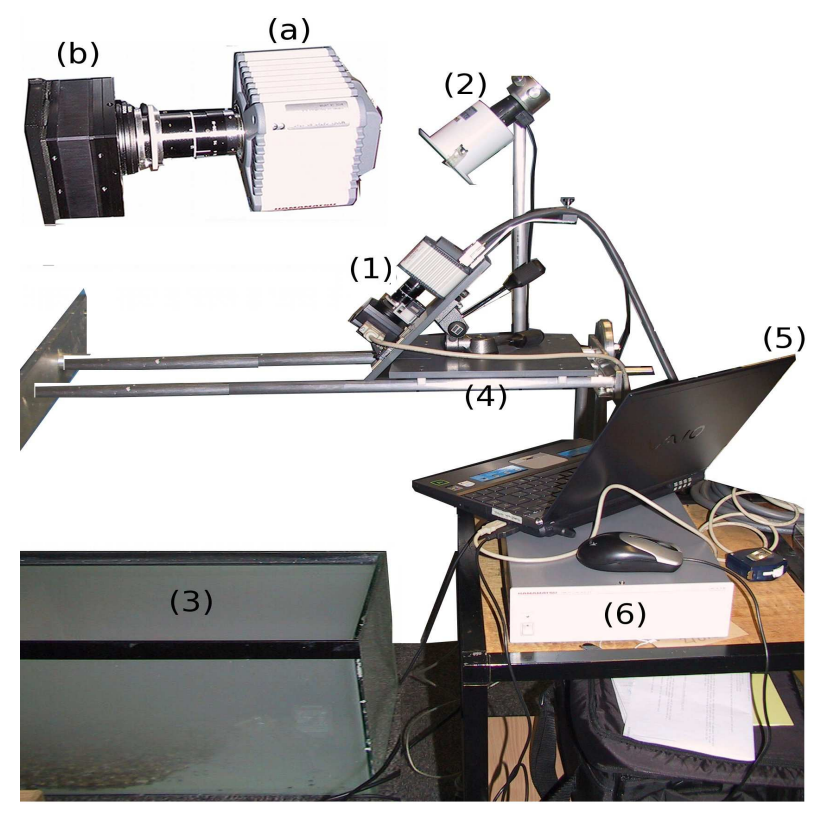

Figure 1. The experimental arrangement for capturing multispectral images. 1-a) Hyperspectral Camera, 1-b) VariSpec optics, 2) Halogen lamp, 3) Water tank, 4) Translation stage, 5) Host computer, 6) The electronics controller module.

A water tank is used to represent underwater medium. A translation stage is designed to translate the camera and light source forward and backward, while the multispectral camera and light source are both set in a specific angle and height relative to the water tank. These conditions are thus similar to those in underwater imaging with an ROV. Figure 1 shows the arrangement of the water tank experiment. The tank is filled with tap water. Absorption by clear tap water is small over the distances involved in this experiment. The dominant degradation to the spectrum is scattering by particles . To represent turbid water, particles in a representative size range are added to the tap water. Emulsion paint, which is mainly composed of Titanium Dioxide $\left(\mathrm{TiO}_{2}\right)$, is used to represent scattering particles. The particles have a diameter in the range 0.2 to $2.5 \mu m$ (Jiang 2004), which is similar to some types of problematic mineral particles in underwater imaging (McCartney 1976). The particles of emulsion paint settle very slowly at the bottom of water tank, providing a constant level of scattering for a limited time. Therefore, the number of sample images should be chosen in such a way that the total capturing time be less than the settling time of particles. 
Hyperspectral imaging system, which consists of a hyperspectral camera and a filter, is used to capture multispectral images. The hyperspectral camera is Hamamatsu ORCA-ER C4742-8012AG, which is a B/W CCD digital camera (Hamamatsu Photonics 2003). The filter is a fast tunable liquid-crystal filter, Varispec, model VIS-10, Cambridge Research \& Instrumentation, Inc., Massachusets (Varispec n.d.), which consists of optics module and electronics controller module. The optics module is mounted in front of the camera and the electronics controller module provides the interface to the host computer. The wavelengths of the light it transmits are electronically controllable, providing rapid, vibrationless selection of any wavelength in the visible (VIS) and near-infrared (NIR) tuning ranges. The filter can be set to $10 \mathrm{~nm}$ or $20 \mathrm{~nm}$ spectral bandwidth. A halogen lamp is used as the light source. The lamp is $75 \mathrm{watt}, 240 \mathrm{~V}$ and made by General Electric (GE) company.

Three series of continuous images, each contains 10 sample images, are captured at different scenes from clear, $T_{0}$, and turbid water conditions, $T_{1}$ and $T_{2}$, at $20^{\circ}$ water temperature, in $10 \mathrm{~nm}$ bandwidth of visible range at $440 \mathrm{~nm}-720 \mathrm{~nm}$ central wavelengths. The images from turbid water are captured at the same camera positions that clear images were captured. Water turbidity at $T_{1}$ and $T_{2}$ are made by adding $0.3 \mathrm{gr} / 100$ Liter $s$ and $0.7 \mathrm{gr} / 100$ Liters of emulsion paint to tap water respectively. The optical distance is $122.08 \mathrm{~cm}$ considering the water refractive index as 1.3334 at water temperature of $20^{\circ}$ (Schiebener et al. 1990) and (Harvey et al. 1998). The camera is set with angle of $\beta 1=42^{\circ}$. The spatial distance between two camera locations for capturing two adjacent images is $4 \mathrm{~mm}$. The camera aperture and focus are set to 4.0 and 1.15 respectively. A halogen lamp is used as the light source and is fixed with angle of $\beta 2=45^{\circ}$. The experiment is done in a dark room with no other source of illumination except the halogen lamp. Water plants, sea shells and gravels are used as scene contents. Due to the relatively long exposure time, compensation is required for the effect of camera dark current noise, the inherent thermal response and the 


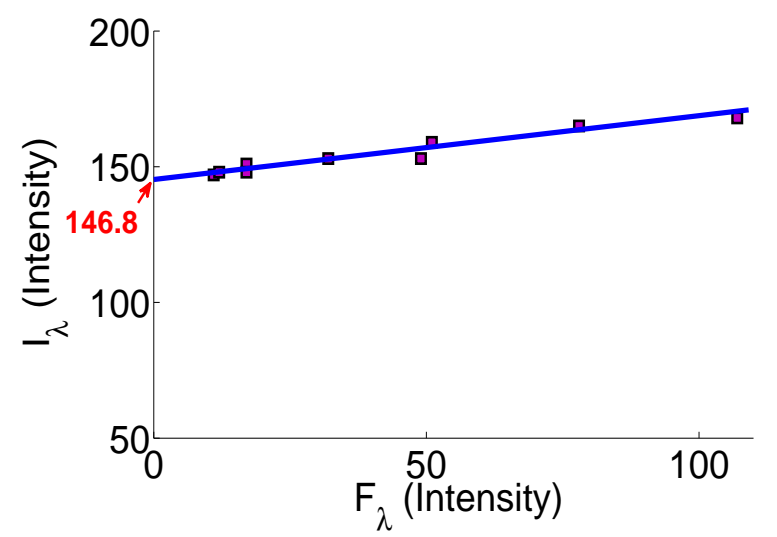

Figure 2. The plot of the degraded versus clear image pixels $\left(I_{\lambda} / F_{\lambda}\right)$. The estimated value of optical backscatter and extinction are about 148.6 and 0.1548 respectively at pixel position $(100,850)$ and $600 \mathrm{~nm}$ wavelength.

fixed pattern noise of the camera at respective wavelengths (Zawada 2003). For that reason a multispectral dark reference is captured according to spectral bands, when the camera is covered completely by a black cloth. The radiance of a white object in clear water is measured with telespectro-radiometer as a reference for the illumination spectrum in clear water. This is used to calculate the reflectance value.

\subsection{Experimental Evaluation: Regression Analysis}

The true values of optical back-scatter and extinction parameters can be estimated by matching the intensity values of degraded image pixels with corresponding clear image pixels from different scenes at a specific wavelength and spatial position. Equation (1) represents the equation of a straight line. Assume that there are two vectors available, where the first one, $I_{\lambda}$, has the intensity values of one pixel position at different scenes of a constant scattering medium and the second one, $F_{\lambda}$, is for the intensity at the same pixel position of the corresponding scenes from a non scattering medium. The regression line (best-fitting line) of the plot of $I_{\lambda}$ versus $F_{\lambda}$ has the slope and Y-intercept of $a_{\lambda}(x, y)$ and $b_{\lambda}(x, y)$ respectively. Figure 2 represents the plot of 10 pixels from images in murky water with respect to images 


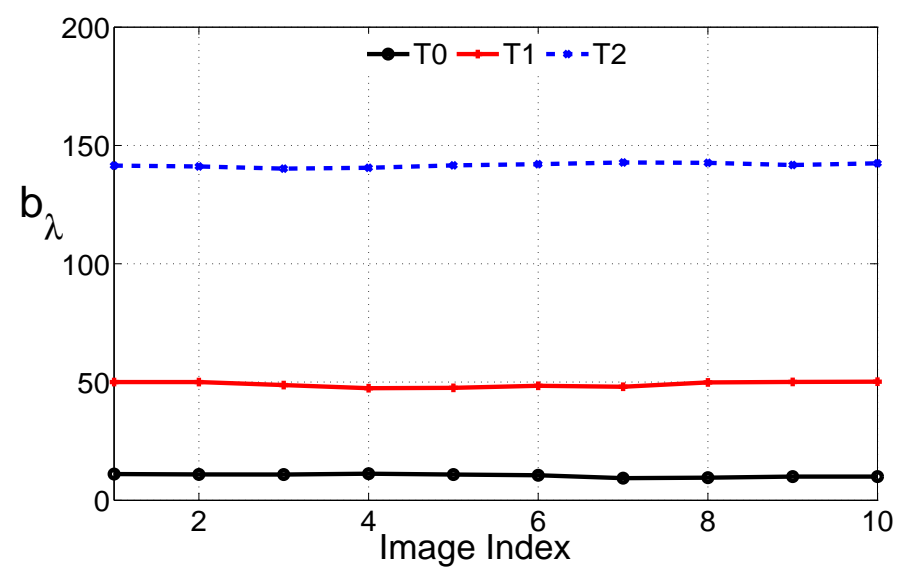

Figure 3. The plot of estimated optical backscatter, $b_{\lambda}$, versus image index (frame number). Optical back-scatter is estimated by extended Oakley-Bu cost function from a sample pixel in spatial position of $(512,672)$, for three water turbidity levels, $T_{0}, T_{1}$ and $T_{2}$ at wavelength of $560 \mathrm{~nm}$.

in clear water at pixel position, $(100,850)$, and at $600 \mathrm{~nm}$ wavelength.

\section{Results}

The actual level of optical back-scatter spectrum for all pixel positions are calculated by regression analysis for two water turbidity levels $T_{1}$ and $T_{2}$ at visible wavelengths of $400 \mathrm{~nm}$ to $720 \mathrm{~nm}$. Optical back-scatter is estimated for 10 degraded images in water turbidity levels $T_{1}$ and $T_{2}$ at respective spectral bands by the simple approach. Optical back-scatter is estimated for 10 degraded images in water turbidity levels $T_{0}, T_{1}$ and $T_{2}$ at respective spectral bands by the proposed method, the extended Oakley-Bu cost function algorithm. The parameters are set to $\sigma_{c f}=4$ and $\sigma_{B}=180$. The algorithm to estimate optical back-scatter is written in Matlab and it requires 6-7 seconds to process an image in each spectral band.

First, the accuracy of the proposed algorithm for optical back-scatter estimation is experimentally investigated. There is always some level of optical back-scatter even for images in clear water condition. Therefore, it is expected that when the proposed algorithm is applied to a clear underwater image, it detects this low level of optical back-scatter. Also, 


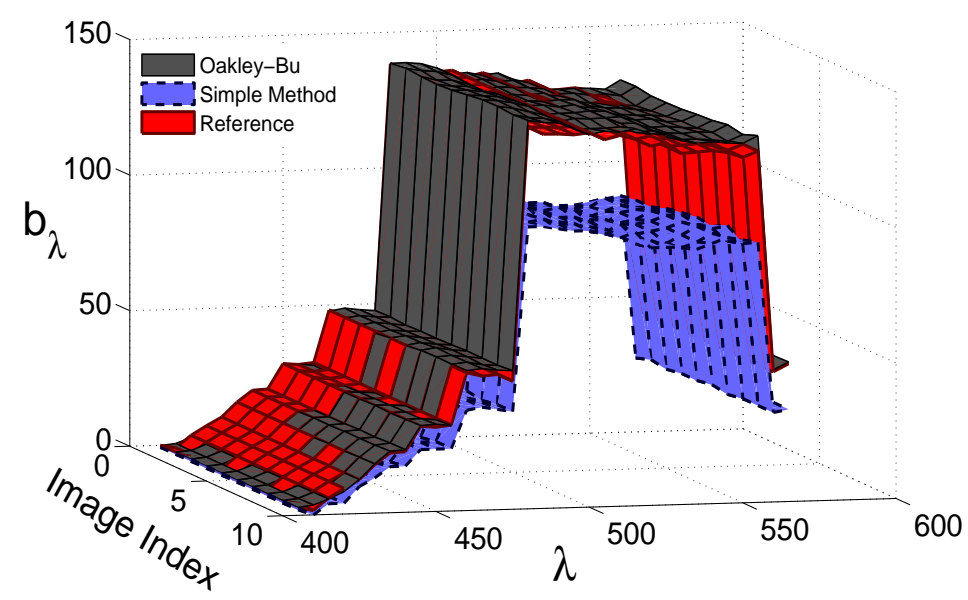

Figure 4. The surface plot of optical backscatter, $b_{\lambda}$, from a sample pixel in a central spatial position of $(512,672)$ for water turbidity level $T_{2}$. The actual value of optical back-scatter found by regression analysis (red), the estimated values by the proposed method, extended Oakley-Bu cost function (grey), the simple method (blue) for 10 images at different spectral bands.

when the level of water turbidity is higher, a higher level of back-scatter is expected. Figure 3 presents the plot of estimated $b_{\lambda}$ for different levels of water turbidity, $T_{0}, T_{1}$ and $T_{2}$, and for 10 images at wavelength of $560 \mathrm{~nm}$. It can be seen from the plot that the value of estimated $b_{\lambda}$ is different for each level and is associated with the level of water turbidity.

Figure 4 presents the surface plot of the actual reference of optical back-scatter (red), the estimated value of optical back-scatter by extended Oakley-Bu cost function (grey) and by the simple method (blue) from a sample pixel in a central spatial position of $(512,672)$ for all wavelengths and for water turbidity level $T_{2}$. It can be seen that the estimated values by the proposed method match the actual reference values to within approximately $+/-2.0 \%$, which confirms the accuracy of the proposed algorithm for optical back-scatter estimation. In contrast the values estimated using the simple method are subject to a negative bias of up to $30 \%$ for some wavelengths. This is partly because the simple method is susceptible to noise and partly because it relies on the presense of a dark object in the image. If the scene has no dark pixels the simple method fails. 

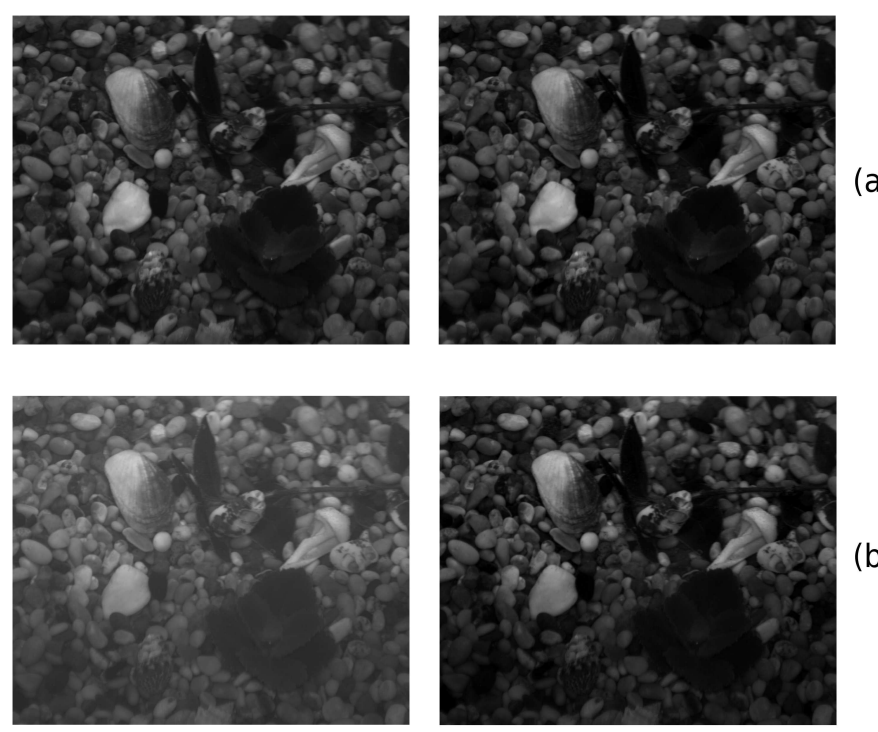

(a)

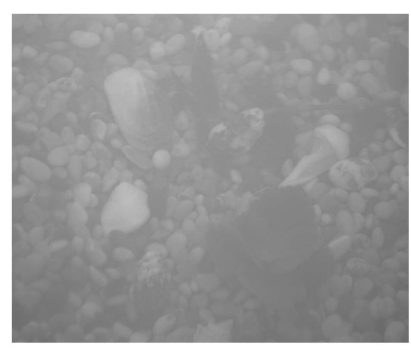

(i)

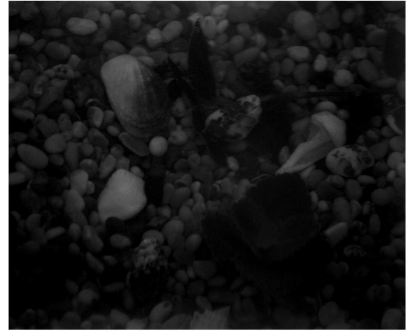

(ii)

(b)

(c)

Figure 5. The selection of i) original images, and ii) improved images at $560 \mathrm{~nm}$ for different levels of water turbidity, a) $T_{0}$, b) $T_{1}$ and $\quad$ c) $T_{2}$ by extended Oakley-Bu cost function.

Next, the consistency of the proposed algorithm for optical back-scatter estimation is analysed. For a series of continuous images, when the level of water turbidity, the optical depth, the illumination and the wavelength remain unchanged, it is expected that the value of optical back-scatter remains unchanged over continuous images. The position of the light source and the water turbidity are kept constant during the capture of 10 test images. As a result, the level of optical back-scatter at each narrow spectral band is expected to be consistent over the 10 test images. It can be seen in Figure 4 that the value of $b_{\lambda}$ is consistent to about 1 grey level for 10 test images at each spectral band. This is true for both the proposed estimation technique and the reference method. 
The estimated values of optical back-scatter are used to recover the degraded image pixels at different levels of water turbidity and different wavelengths. The scaling for the recovered image is done by stretching the image histogram. A selection of improved images at $560 \mathrm{~nm}$ is presented in Figure 5. It can be seen that the improved images from $T_{0}$ and $T_{1}$ (Figures 5(a) and (b) at column (ii)) are similar. The improved images show the scene content without any scattering effect. This confirms that the algorithm can detect the level of optical back-scatter and correct the image appropriately.

Figure 6 shows the spectra for three specific pixels corresponding to three different surface types, (a) sea shell, (b) water plant and (c) gravel. The blue traces show the uncorrected spectral intensities on a scale between 0.0 and 1.0, where 1.0 represents maximum intensity. It can be seen that the uncorrected spectral intensities are very similar for all three surfaces. This is because most of the light in the multispectral images is due to scattering from particles; only a small component is due to surface reflection. Hence these spectra are mainly determined by the scattering properties of the particles and the spectrum of the illumination. The black traces show the spectral intensity in the case of clear water. These spectra show significant differences as might be expected for such different surfaces. The red traces show spectral reflectance estimated using the technique described in this paper. It is clear that the level of similarity between the estimated reflectance and the spectral intensity for clear water is very much higher than uncorrected spectral intensity and the spectral intensity for clear water. The match is less good at some shorter wavelengths. This is because the level of noise is higher in shorter wavelengths. These data are provided as an illustration of the potential of the technique. Future study is required to quantify the accuracy of the recovered reflectance as a function of wavelength. 


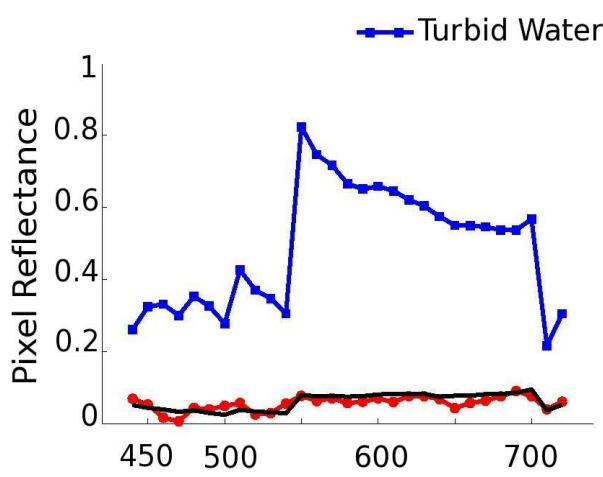

(a)

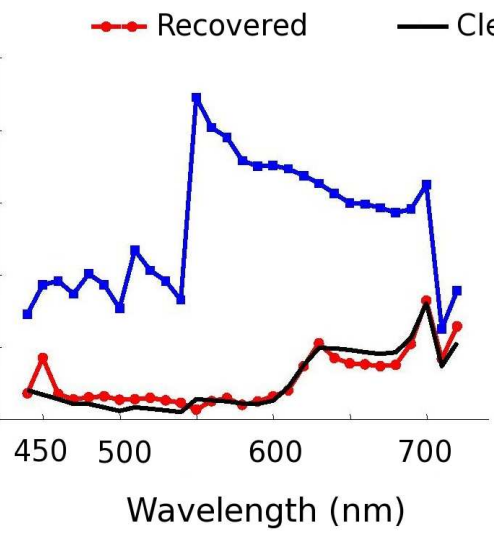

(b)

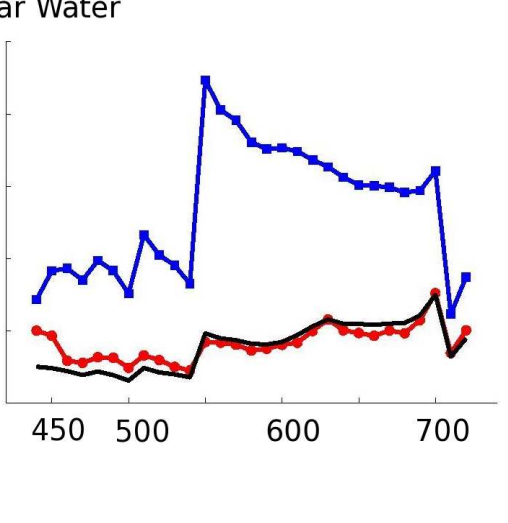

(c)

Figure 6. The spectral reflectances plot of image index 1 from the recovered pixel, the correspondence in clear and turbid water conditions $\left(T_{2}\right)$ for three different objects, a) gravel $(100,850)$ b) water plant $(840,350)$ c) sea shell $(605,805)$.

\section{Conclusion}

The main problem in underwater imaging is the effect of optical back-scatter changes image intensity and leads to inaccuracy in the pixel spectra. The experimental results show that a simple approach fails to accurately estimate the level of optical back-scatter. Furthermore, the proposed method can produce an accurate estimate of optical back-scatter at different spectral bands in visible wavelengths and for different levels of water turbidity. The estimated optical back-scatter is consistent over continuous images with same level of optical backscatter. By subtracting the backscatter, more accurate pixel spectra are obtained. However, for very high level of turbid water the recovery is limited because of the effect of extinction. The advantage of the proposed method is that it compensates the effect of optical back-scatter with no information of the physical properties of the medium. Only a multispectral image is required.

\section{Acknowledgment}

I would like to thank Prof. Foster for his valuable discussions and for providing the facility for multispectral imaging and also Dr. Kinjiro Amano for his assistance in capturing multispectral 
images.

\section{Appendix A. Oakley \& Bu cost function with variable offset}

The original Oakley \& Bu cost function, which is presented by (A.1), assumes optical backscatter has a constant value, $b_{\lambda}$.

$$
S(\kappa)=\frac{1}{P} \sum_{j=1}^{P}\left(\frac{I_{j}-\bar{I}_{j}}{\bar{I}_{j}-b_{\lambda}}\right)^{2} \cdot \exp \frac{1}{P} \sum_{j=1}^{P} \ln \left(\bar{I}_{j}-b_{\lambda}\right)^{2} .
$$

A synthetic model of optical back-scatter in underwater is required to demonstrate the algorithm of Oakley-Bu cost function can be extended for variable back-scatter. From the study of several real continuous underwater images (Mortazavi 2006), it is found that the spatial distribution of light intensity can be simulated by a positive truncated cosine function. In addition, the spatial distribution of an optical back-scatter function follows the spatial distribution of light variation across the image. As a result, the optical back-scatter function, $b_{\text {syn }}(\lambda)$, can be simulated by (A.2).

$$
b_{\text {syn }}(\lambda)=\gamma_{s} \cos (\theta) S b_{\text {syn }}(\lambda), \quad \frac{-\pi}{2}<\theta<\frac{\pi}{2}
$$

where $\gamma_{s}$ is the parameter used to set the level of optical back-scatter intensity, $\cos (\theta)$ is the spatial distribution of the light intensity across an image row, and $S b_{\text {syn }}(\lambda)$ is the optical backscatter spectrum. The optical back-scatter function (A.2) is substituted with $b_{\lambda}$ in (A.1) with $S b_{\text {syn }}(\lambda)=1$ and $\gamma_{s}=\kappa$ :

$$
S(\kappa)=\frac{1}{P} \sum_{j=1}^{P}\left(\frac{I_{j}-\bar{I}_{j}}{\bar{I}_{j}-\kappa \cos (\theta)}\right)^{2} \cdot \exp \frac{1}{P} \sum_{j=1}^{P} \ln \left(\bar{I}_{j}-\kappa \cos (\theta)\right)^{2} .
$$

In recent study, (Bu 2007), it is proved that the first derivative of $S(b)$ has minimum value when $b=c$.

$$
\left.\frac{d(S)}{d(b)}\right|_{b=c}=0 \text {. }
$$

For parametric offset, again we are looking for the value of offset function which makes the cost function minimum. The first derivative of the $S(\kappa)$ by using the chain rule is given by:

$$
\frac{d(S)}{d(\kappa)}=\frac{d(S)}{d(b)} \frac{d(b)}{d(\kappa)}
$$

From (A.2):

$$
\frac{d(b)}{d(\kappa)}=\cos (\theta)
$$

Substituting (A.6) in (A.5) gives:

$$
\frac{d(S)}{d(\kappa)}=\frac{d(S)}{d(b)} \cos (\theta)
$$

$\frac{d(S)}{d(\kappa)}$ is zero when either $\frac{d(S)}{d(b)}=0$ or $\cos (\theta)=0$. The range of $\theta$ is defined in (A.2) and as a result $\cos (\theta)$ never has zero value. Therefore, $\frac{d(S)}{d(b)}=0$ which shows the function can have a minimum value at the offset value. 


\section{References}

Bu H 2007 Digital Image Enhancement Phd Electrical and Electronic Eng. The University of Manchester.

Caimi F M, Kocak D M, Dalgleish F \& Watson J 2010 IEEE Oceanic Engineering Society Newsletter pp. 21-29.

Dahms H U \& Hwang J S 2010 Journal of Marine Science and Technology 18(1), 112-121.

Fan T Y, Jan R Q \& andChin Ho Hsu C P L 2008 Monthly Journal of Taipower's Engineering 721(9), 96-103. Foresti G 2001 IEEE Trans. Syst.Man and Cyber part B 31, 691-705.

Gleason A C R, Reid R P \& Voss K J 2007 in 'Oceans 2007’ Oceans MTS/IEEE Conference.

Hamamatsu Photonics 2003.

URL: http://sales.hamamatsu.com/assets/pdf/hpspdf/C4742-95-12ER.pdf

Harvey A H, Gallagher J \& Sengers J 1998 Journal of Physical and Chemical Reference Data 27(4), 761-74.

Holden H \& LeDrew E 2002 Remote Sensing of Environment 81(2-3), 300-308.

Jiang D 2004 Digital image enhancement Mphil Electrical and Electronics Eng.

Kondo H \& Ura T 2004 Control Engineering Practice 12(12), 1551-1559.

Lubin D, Li W, Dustan P, Mazel C H \& Stamnes K 2001 Remote Sensing of Environment 75(1), 127137.

McCartney E J 1976 Optics of the atmosphere scattering by molecules and particles Wiley.

Mortazavi H 2006 Underwater image enhancement Mphil Electrical and Electronics Eng.

Mortazavi H 2010 Mitigation of Contrast Loss in Underwater Images Phd Electrical and Electronics Eng.

Mortazavi H \& Oakley J 2007 in 'ICMSAO2007'.

Mukherjee K, Gupta S \& Phoha S 2011 IEEE Journal of Oceanic Engineering 36(2), 219-230.

Oakley J \& Bu H 2007 IEEE, Trans. on Image Processing 16(2), 511-522.

Peddle D R, White H P, Soffer R J, Miller J R \& LeDrew E F 2001 Computers and Geosciences 27, 203213.

Rao C, Mukherjee K, Gupta S, Ray A \& Phoha S 2009 in ‘ACC 2009' American Control Conference.

Schechner Y \& Karpel N 2005 IEEE J. of Oceanic Eng. 30(3), 570-587.

Schechner Y Y \& Averbuch Y 2007 IEEE Trans. on Pattern Analysis and Machine Intelligence 29(9), 16551660.

Schechner Y Y \& Karpel N 2004 in 'CVPR 04' Vol. 1 Proc. of the 2004 IEEE Computer Soc. Conf. on Computer Vision and Pattern Recognition pp. 536-43.

Schiebener P, Straub J, Sengers J L \& Gallagher J 1990 Journal of Physical and Chemical Reference Data 19(3), 677-717.

Sun H, Benzie P W, Burns N, Hendry D, Player M \& Watson J 2008 Philosophical Transactions of the Royal Society A: Mathematical, Physical and Engineering Sciences 366(1871), 1789-1806.

Varispec n.d.

URL: http://www.spectralcameras.com/varispec

Whitmire A L, Pegau W, Karp-Boss L, Boss E \& Cowles T J 2010 Optics Express 18(14).

Zawada D G 2003 IEEE, Journal of Oceanic Engineering 28(4), 583-594. 